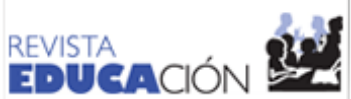

Revista Educación

ISSN: 0379-7082

ISSN: 2215-2644

revedu@gmail.com

Universidad de Costa Rica

Costa Rica

\section{Diseño de un modelo universitario de vinculación laboral y apoyo a la gestión académica}

\author{
González Conejo, Franky \\ Diseño de un modelo universitario de vinculación laboral y apoyo a la gestión académica \\ Revista Educación, vol. 45, núm. 2, 2021 \\ Universidad de Costa Rica, Costa Rica \\ Disponible en: https://www.redalyc.org/articulo.oa?id=44066178014 \\ DOl: https://doi.org/10.15517/revedu.v45i1.43083
}

\section{(c) (1) 90}

Esta obra está bajo una Licencia Creative Commons Atribución-NoComercial-SinDerivar 3.0 Internacional. 


\section{Diseño de un modelo universitario de vinculación laboral y apoyo a la gestión académica}

\section{Design of a university model of labor linkage and support for academic management}

Franky González Conejo

Universidad Nacional, Costa Rica

fgonzale@una.cr

(iD) https://orcid.org/0000-0002-9991-8078

\author{
DOI: https://doi.org/10.15517/revedu.v45i1.43083 \\ Redalyc: https://www.redalyc.org/articulo.oa? \\ $\mathrm{id}=44066178014$
}

Recepción: 28 Julio 2020

Aprobación: 05 Octubre 2020

\section{RESUMEN:}

El presente ensayo tiene como propósito plantear el diseño de modelo universitario de vinculación laboral, resultante de la reflexión posterior a la investigación positivista desarrollada por el autor, basada en el estudio de cuatro casos institucionales; para esto se analizan los aspectos relevantes acerca de los servicios de empleo en las universidades estatales costarricenses, las condiciones del mercado laboral profesional y los referentes teóricos más importantes, especialmente desde la perspectiva teórico-práctica de la Orientación Laboral. Se estima que esta propuesta de modelo podría tener efectos positivos en el abordaje de las incertidumbres que enfrentan las personas universitarias ante la desmesurada sobreoferta de profesionales, el posible desfasaje con respecto a los requerimientos del mundo del trabajo que presenta indicadores cada vez menos favorables, lo que asegura la insuficiencia de una sola disciplina para investigar e intervenir el fenómeno de inserción laboral, así como la impostergable vinculación entre el sector empleador y la academia universitaria; por ello, podría constituir un insumo valioso para las demás universidades, principalmente de Latinoamérica, debido a la similitud contextual, favoreciendo sus procesos de asesoramiento para la inserción laboral del estudiantado universitario y las personas graduadas, así como los procesos de gestión de la calidad de las carreras.

Palabras Clave: Educación, Universidad, Educación y empleo, Asesoramiento, Orientación Profesional.

\section{Abstract:}

This essay aims at designing a university employment model. The author analyzes four state universities and job opportunities for its graduates through theoretical-practical perspectives and career guidance. It is estimated that this proposed model could have positive effects in the approach of the uncertainties that university students face due to increasing oversupply of professionals. A possible disparity with the requirements of the labor market reflect less favorable indicators which ensures a lack of a single discipline in order to research or intervene the phenomenon labor insertion, as well as the unavoidable bond between the employment sector and the university. The proposed model could, therefore, serve as valuable input for other academic institutions, mainly Latin American universities given the contextual similarity which also strives to improve career guidance for its university students and graduates and provide a quality management process for its majors.

KEYWORDS: Education, University, Education and Employment, Counseling, Career Guidance.

\section{INTRODUCCIÓN}

El problema del desempleo es una lacra social que siempre ha existido en todo el mundo y es el culpable mayoritariamente del detrimento económico de las personas, de las familias que dependen de los ingresos de dichas personas, y de las sociedades que tienen a esas familias como base fundamental.

Este problema global, lejos de mejorar, pareciera que se agrava con el pasar de los años y afecta a todos los sectores sociales y productivos, de los cuales se podría pensar que las personas profesionales que se han titulado de las universidades no sufren de tal situación, o al menos con el mismo grado que otras personas que no forman parte de dicha élite intelectual y socioeconómica del país, sin embargo los indicadores de inserción laboral de este grupo han venido desmejorando en los últimos años, como se describe en este ensayo, apoyado por los últimos datos del Instituto Nacional de Estadística y Censo [INEC] (2020), del IIV 
Informe de Estado de la Educación (Programa Estado de la Nación, 2019) y los estudios de seguimiento que se han efectuado principalmente por Gutiérrez, Kikut, Corrales y Picado (2018) a esta población desde el Observatorio Laboral de Profesiones del Consejo Nacional de Rectores (OLaP-CONARE), así como una revisión crítica desde otros autores e instancias que aportan al análisis del mercado de trabajo profesional.

Asimismo, se hace un acercamiento teórico a la concepción de Servicio Público de Empleo que aporta De Pablo (2011) y los principales referentes en el ámbito universitario estatal de nuestro país, como el Consejo Nacional de Rectores y el Sistema Nacional de Acreditación [SINAES] (2009), de donde se desprende el diseño de un modelo de vinculación laboral, fundamentado especialmente desde la perspectiva teórica de la Orientación Laboral que aportan Echeverría, Isus, Martínez y Sarasola (2008), Barreto (2016) y el Sistema de Intermediación, Orientación e Información de Empleo [SIOIE] (2010b), así como el propio autor (González, 2019), desde su formación y experticia profesional.

Por consiguiente, el presente ensayo tiene como propósito difundir el modelo diseñado por el autor, resultante de la reflexión académica y la investigación desarrollada en el ámbito universitario estatal costarricense, que podría constituirse en un insumo importante en el favorecimiento de los procesos de gestión de carreras en cuanto a su vinculación con el sector empleador y de inserción laboral del estudiantado y personas graduadas de las universidades, cuyos contextos puedan tener alguna similitud al costarricense, como podría ser el caso de los países de América Latina, aspectos que se relacionan estrechamente con la calidad y pertinencia de la educación, sobre todo en cuanto a procesos de autoevaluación, acreditación y mejoramiento se refiere.

De igual manera, la propuesta que se presenta eventualmente podría fortalecer algunas líneas de la praxis cotidiana en los servicios de empleo en las universidades, especialmente desde el abordaje integral y estratégico que requiere la articulación de estas instancias, con otras dependencias universitarias como son las propias unidades académicas a las que pertenecen las carreras.

\section{Los Servicios Públicos de Empleo}

Como parte de la revisión documental y como primer antecedente práctico para la elaboración de la presente investigación, es necesario, en primera instancia, familiarizarse con la figura de los Servicios Públicos de Empleo (SPE), los cuales según De Pablo (2011) se conciben en el contexto centroamericano como un modelo de intervención desarrollado en las instituciones públicas o estatales, para apoyar la inserción o reinserción de personas adultas al mercado laboral.

En el ámbito nacional costarricense, en el año 2000 se crea el Sistema Nacional de Intermediación, Orientación e Información de Empleo [SIOIE] (2010a), que consiste en un modelo plurinstitucional con participación directa del Ministerio de Trabajo y Seguridad Social (MTSS), el Instituto Nacional de Aprendizaje (INA) y el Ministerio de Educación Pública (MEP), además de los gobiernos locales que cuentan con bolsa de empleo, utilizando una plataforma que vincula oferta y demanda laboral, así como una guía metodológica para las personas Gestoras de Empleo.

Recientemente, bajo la administración del actual Presidente de la República Carlos Alvarado Quesada, se establece el nuevo proyecto de la Agencia Nacional de Empleo como "la instancia técnica y operativa que articula los servicios de empleo, la red de unidades de empleo, la plataforma informática" (Agencia Nacional de Empleo, 2020, pár. 1), la cual está adscrita al Sistema Nacional de Empleo, anterior SIOIE. Aún es un poco prematuro para adelantar criterio con respecto a la pertinencia de transformar una instancia de tales dimensiones y que se enfoca en favorecer uno de los temas más importantes para un país como es el empleo.

De manera específica, en cuanto a los servicios de empleo en el ámbito universitario, en Costa Rica las universidades estatales han emprendido importantes esfuerzos para atender la necesidad que tiene el estudiantado y las personas graduadas de recibir algún apoyo en el proceso de transición del estudio superior al mundo del trabajo, así como los requerimientos de talento profesional del sector empleador, aspectos 
que fueron abordados por González (2019), y de los cuales se presentan en la Tabla 1 algunos elementos importantes del contexto institucional, del enfoque y los objetivos de los servicios de empleo, exceptuando la Universidad Nacional (UNA) por razones de subjetivación ${ }^{[1]}$ para diseñar la propuesta que se detalla en este trabajo.

TABLA 1.

Resumen comparativo de los elementos más importantes de los servicios de empleo en cuatro de las universidades estatales costarricenses.

\begin{tabular}{|c|c|c|c|c|}
\hline $\begin{array}{l}\text { Aspectos } \\
\text { del contexto } \\
\text { institucional }\end{array}$ & $\begin{array}{l}\text { Talento TEC, Instituto } \\
\text { Tecnológico de Costa Rica } \\
\text { (ITCR) }\end{array}$ & $\begin{array}{l}\text { Servicio de Intermediación } \\
\text { Laboral, Universidad de } \\
\text { Costa Rica (UCR) }\end{array}$ & $\begin{array}{l}\text { Bolsa de Empleo, Universidad } \\
\text { Estatal a Distancia (UNED) }\end{array}$ & $\begin{array}{l}\text { Sistema de Intermediación de } \\
\text { Empleo, Universidad Técnica } \\
\text { Nacional (UTN) }\end{array}$ \\
\hline $\begin{array}{l}\text { Año de } \\
\text { creación e } \\
\text { instancia de } \\
\text { adscripción }\end{array}$ & $\begin{array}{l}2008 \text {, Centro de } \\
\text { Vinculación es una unidad } \\
\text { académica no docente de la } \\
\text { Vicerrectoria de Extensión. }\end{array}$ & $\begin{array}{l}2008 \text {, Centro de Orientación } \\
\text { Vocacional-Ocupacional es } \\
\text { una unidad operativa se } \\
\text { especializa de la Oficina de } \\
\text { Orientación en la } \\
\text { Vicerrectoria de Vida } \\
\text { Estudiantil. }\end{array}$ & $\begin{array}{l}\text { 2010, Cátedra de Recursos } \\
\text { Humanos de la Escuela de } \\
\text { Ciencias de la Administración, } \\
\text { que es una unidad académica. }\end{array}$ & $\begin{array}{l}\text { 2011, Área de Bienestar Estudiantil } \\
\text { de la Vicerrectoria de Vida Estudiantil. }\end{array}$ \\
\hline $\begin{array}{l}\text { Naturaleza } \\
\text { del servicio } \\
\text { o enfoque }\end{array}$ & $\begin{array}{l}\text { Programa que actúa como } \\
\text { bolsa de empleo virtual. }\end{array}$ & $\begin{array}{l}\text { Servicio de asesoria, } \\
\text { componente del eje de } \\
\text { desarrollo vocacional- } \\
\text { ocupacional, que realiza } \\
\text { intermediación. }\end{array}$ & $\begin{array}{l}\text { Servicio de bolsa de empleo de } \\
\text { rango institucional. }\end{array}$ & $\begin{array}{l}\text { Servicio de bolsa de empleo que } \\
\text { realiza intermediación laboral. }\end{array}$ \\
\hline $\begin{array}{l}\text { Extracto de } \\
\text { los objetivos }\end{array}$ & $\begin{array}{l}\text { Es un espacio "bilateral" } \\
\text { para estudiantes, personas } \\
\text { graduadas y sector } \\
\text { empresarial. }\end{array}$ & $\begin{array}{l}\text { Asesorar el estudiantado } \\
\text { próximo a graduarse en su } \\
\text { preparación para el mundo } \\
\text { laboral. }\end{array}$ & $\begin{array}{l}\text { Acompañar a estudiantes y } \\
\text { personas graduadas en su } \\
\text { inserción laboral. }\end{array}$ & $\begin{array}{l}\text { Vincular entre estudiantes, personas } \\
\text { graduadas, sectores productivos y } \\
\text { organizaciones para promover la } \\
\text { empleabilidad y trabajo digno. }\end{array}$ \\
\hline
\end{tabular}

Fuente: elaborada a partir de González (2019).

De los elementos resumidos en la Tabla 1, y la investigación realizada para este trabajo, se rescata que los servicios de empleo en las universidades costarricenses son de reciente data, situación que podría justificar la escasa cantidad de referencias de investigaciones enfocadas en su praxis, razón por la cual toma mayor valor teórico y metodológico el estudio de González (2019), y la posterior profundización con el presente ensayo. Además, es significativo resaltar que la mayoría de los modelos estudiados corresponden a sistemas de bolsa de empleo, enfocados en favorecer la empleabilidad de las personas estudiantes y graduadas, en tanto que solamente una de ellas realiza vinculación con el sector productivo de manera estratégica, sin embargo, dicha relación no permea la gestión académica de las carreras.

González (2019) además recomienda a los servicios de empleo estudiados, la posibilidad de incorporar esfuerzos de investigación o mapeo de los indicadores más relevantes del trabajo y de la condición laboral de sus profesionales. Dichos indicadores (como el desempleo, el subempleo, la tasa de presión general, la relación entre el trabajo y la carrera, entre otros) se estiman indispensables para facilitar el asesoramiento a estudiantes, personas graduadas y a las propias instancias universitarias encargadas de gestionar las carreras y demás procesos institucionales de toma de decisiones, como se describe más adelante.

\section{CONDICIONES DEL MERCADO LABORAL PROFESIONAL}

Particularmente, con respecto al desempleo, la Organización Internacional del Trabajo [OIT] (2020) prevé que aumente de manera considerable a nivel mundial entre 2020 y 2022, pero lo más grave es que la desigualdad de ingresos será mayor de lo que se pensaba, debido a una elevada precariedad laboral y una creciente tasa de presión general, así como un gran número de personas que aún no pueden buscar empleo 
o han dejado de hacerlo, lo que suma casi quinientos millones de personas infrautilizadas y que su potencial no es aprovechado laboralmente. $Y$ es que, en un mundo globalizado e interconectado gracias a los avances tecnológicos, pero plagado a la vez de desigualdades socioeconómicas persistentes y de acceso a la educación y al trabajo, el valor que se otorga a este último es central, no solamente para la autorrealización de cada persona, sino también para el bienestar de la familia como base de toda sociedad (Rivas, 2003).

Costa Rica no escapa de reflejar esta realidad contextual, particularmente porque el desempleo es un fenómeno multicausal, que siempre figura en los programas de candidaturas presidenciales y en las agendas de gobierno, el cual lejos de mejorar, sigue manteniendo la tendencia mundial con altos índices, a pesar de los esfuerzos que desarrollan diferentes sectores (opuestos en algunas ocasiones) como el gubernamental y el productivo.

Recientemente el INEC (2020) determinó que, para el I trimestre de 2020, la tasa de desempleo llegó a 12,5\%, aumentando 1,2 puntos porcentuales con respecto al año anterior, y el subempleo subió 3,8\% para llegar a $12,4 \%$, en tanto que la tasa de presión general crece 3 puntos alcanzando un $22,4 \%$ de forma interanual. A pesar de que se tienen indicadores más positivos que en la mayoría de los países afectados por la pandemia del COVID-19, las expectativas de que el panorama socioeconómico empeore son muy altas en nuestro país, por lo que, ante esta situación, se podría pensar que las personas con estudios universitarios como grupo élite, se ven exentas de todas estas problemáticas a la hora de buscar trabajo, y, si bien es cierto que la formación superior favorece las posibilidades laborales, como lo demuestran los estudios de seguimiento a personas graduadas universitarias, estas mismas investigaciones revelan que el sector profesional no se exime de esta realidad y que refleja los mismos indicadores, aunque ciertamente en menor medida.

$\mathrm{Al}$ respecto, Gutiérrez et al. (2018), determinaron en su último estudio para el Observatorio Laboral de Profesiones del Consejo Nacional de Rectores (OLaP-CONARE), que la tasa de desempleo luego de 3 a 5 años de titularse de la universidad, aumentó en cada uno de los estudios anteriores para llegar al 5,6\%, siendo más alta en las profesionales mujeres con 7,5\%, repitiendo el patrón de asimetría que se da en otros sectores laborales, así como a nivel nacional, latinoamericano y mundial. Asimismo, el desempleo se acentúa en áreas como Ciencias Sociales, con 9,0\%, Ciencias Básicas y Ciencias de la Salud con 7,1\% y Recursos Naturales con 7,0\%; particularmente destaca que el desempleo en 17 disciplinas sobrepasa el 9,5\%, reportado por el INEC (2016) en el mismo periodo a nivel nacional para toda la población costarricense.

En el caso de las y los profesionales que lograron insertarse laboralmente, también pueden verse afectados por un mercado laboral donde la oferta sobrepasa la demanda, experimentando subempleo por insuficiencia de horas, el cual tuvo un aumento considerable, pasando de 3,1\% a 5,1\%, situación que se replicó en casi todas las áreas de conocimiento (Gutiérrez et al., 2018). Además, el 7,5\% de las personas consultadas en dicho estudio consideran que su trabajo tiene poca o ninguna relación con su carrera, factor que tuvo un importante incremento con respecto al estudio anterior, esto implica un aumento en la cantidad de personas que no pueden ejercer su profesión para la cual dedicaron tanto tiempo, esfuerzo y dinero propio y de su grupo familiar, por lo que debido a las dificultades para encontrar empleo relacionado con su perfil profesional en las zonas rurales, una gran parte de esta fuerza laboral opta por quedarse en la Región Central del país, por la diversidad de espacios de trabajo y estudio, principalmente en la provincia de San José. Por supuesto que tal escenario no contribuye al desarrollo socioeconómico de las zonas periféricas del territorio nacional, a pesar de la presencia de diversas sedes y campus universitarios en todo el territorio nacional.

\section{APUNTES UNIVERSITARIOS PARA LA VINCULACIÓN LABORAL}

El nivel que ha alcanzado la brecha entre oferta y demanda laboral también afecta y genera incertidumbre en las personas graduadas universitarias, debido principalmente a la incapacidad del Estado y el sector privado de crear nuevos puestos para empleos calificados y a la desmesurada sobreoferta de profesionales, muestra de 
ello es que entre 2012 y 2017 el promedio anual de entrega de títulos universitarios fue de 46.800, según el VII Informe del Estado de la Educación (Programa Estado de la Nación, 2019).

Toda esta situación contextual de competitividad e incertidumbre pone mucha presión sobre los hombros de las y los universitarios, principalmente de quienes están cerca de graduarse y buscar ese primer trabajo tan idealizado, presentándose un desajuste o desfasaje entre el perfil profesional de la persona y los requerimientos del ámbito laboral donde desea insertarse, esto desde la perspectiva del Sistema Nacional de Acreditación de la Educación Superior (SINAES, 2009) y de autores como Teichler (2005), Enriques (2007), Echeverría et al. (2008).

Lo anterior es muestra de la necesidad de vincular el sistema universitario con el sector productivo, como sugiere en una entrevista Thomas Wissing, Director Adjunto de la OIT y Representante para México y Cuba, para potenciar oportunidades de trabajo que prevenga en las personas egresadas no solamente las elevadas tasas de desempleo en todo el mundo, sino que además evite que se hundan en la informalidad laboral o la necesidad de emigrar (Muñoz, 2012).

Desde la perspectiva de Orientación Laboral que sustenta Barreto (2016), resulta estratégico para los servicios universitarios de empleo, y para las mismas universidades el fortalecer alianzas con las organizaciones empleadoras de sus profesionales, en virtud del diálogo y el trabajo colaborativo interdisciplinar e intersectorial, que potencie las experiencias laborales del estudiantado y la inserción de las personas graduadas.

En la misma línea, la Conferencia Regional de Educación Superior (CRES), coordinada por el Instituto Internacional de la UNESCO para la Educación Superior de América Latina y el Caribe [IESALC] (2018), en el Plan de Acción 2018-2028 hace un vehemente llamado a todas las universidades para "Diseñar y fortalecer sistemas de seguimiento de personas graduadas que permitan evaluar la calidad de la formación en las IES, identificando requerimientos en áreas estratégicas para el desarrollo científico, social y cultural sostenible de nuestros países" (p. 37).

$\mathrm{Al}$ respecto, se considera impostergable iniciar con algunas líneas de coordinación o vinculación entre las universidades y el sector empleador, que favorezca no solamente la inserción laboral, sino que además facilite la realimentación de la oferta docente, especialmente el diseño, rediseño y gestión de los planes de estudio, desactualizados o con insuficiencia de las competencias necesarias en la actualidad y que no se exigían en el momento en que se diseñaron, como es el caso de las relacionadas con la autogestión y la comunicación, las más valoradas por el sector empleador, pero también las que evidencian mayor brecha de formación, como se estableció en el Estudio de Empleadores 2016 del OLaP- CONARE (Gutiérrez, Madrigal, Corrales y Kikut, 2019).

\section{DiSEÑo DE UN MODELO UNIVERSITARIO DE VINCULACIÓN LABORAL}

Es necesario, en el aquí y el ahora, que se aproveche el camino recorrido, y que se integren, desde una visión más amplia y sistémica, los intereses, los esfuerzos y los recursos, para poder ofrecer a este dilema complejo, una solución compleja. 


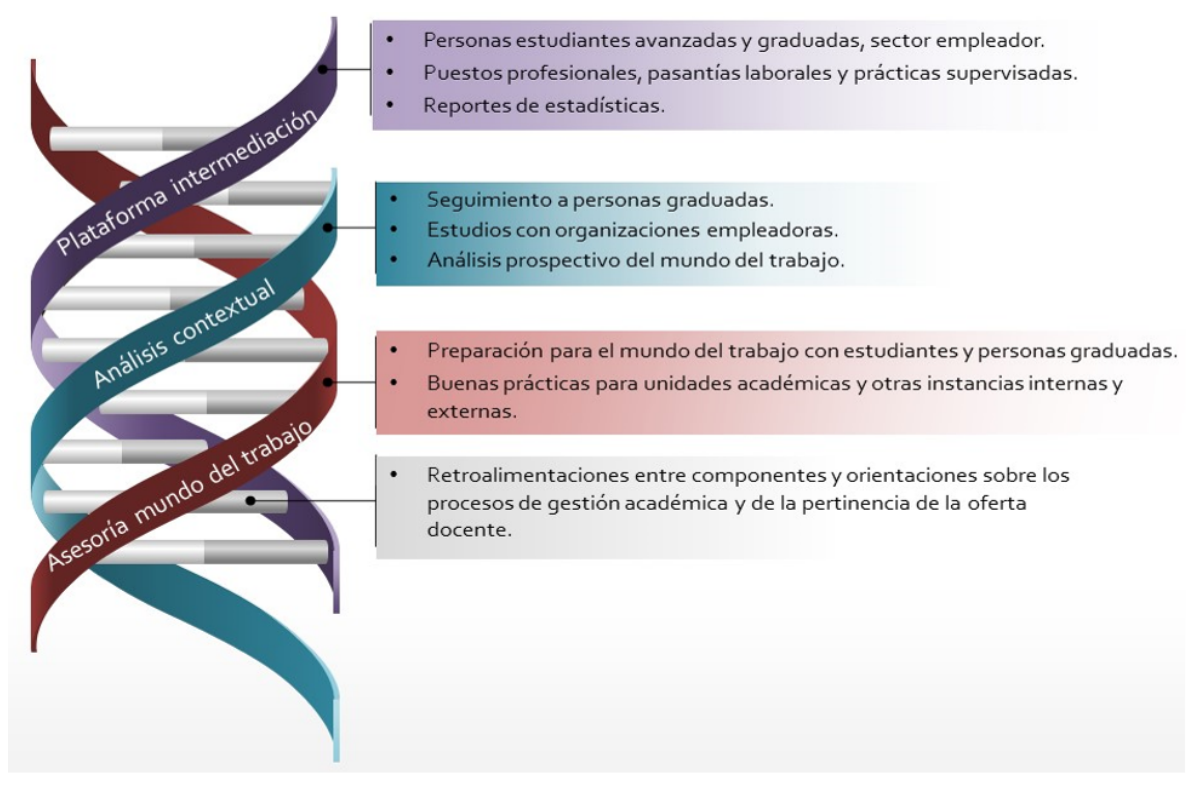

FIGURA 1.

Modelo Universitario de Vinculación Laboral

Fuente: González (2019).

Con fundamentación en los elementos que se exponen en este ensayo, el autor profundiza por primera vez en el diseño de un modelo de vinculación laboral correspondiente a un sistema compuesto de partes interconectadas que busca insertar en el ADN universitario la vinculación permanente con las personas graduadas y el sector empleador, como se muestra en la Figura 1.

De las interacciones que se dan entre sus tres componentes y la forma en que se complementan y se nutren uno de otro, se desprende la siguiente descripción de cada uno de ellos.

a) Plataforma de intermediación laboral

Mediante una plataforma informática y desde la perspectiva de Mazza (2003), la universidad puede intermediar o vincular la oferta de currículum vitae de las personas profesionales o avanzadas en sus estudios y la demanda de talento cualificado de las organizaciones empleadoras, a la vez que facilita asesoría para la inserción al mundo del trabajo mediante sesiones grupales y el desarrollo de productos multimedia.

El rol de empresa o institución empleadora permite publicar anuncios de puestos profesionales u oportunidades de pasantías laborales que faciliten la adquisición de experiencia y otros aprendizajes al estudiantado en ejercicio de su disciplina, así como opciones para desarrollar práctica supervisada en el último semestre como requisito para titularse de grado. Las instancias universitarias, unidades académicas y carreras, tendrían un rol de administrador que les permita consultar reportes de estadísticas y visualizar los perfiles de las personas candidatas o de las organizaciones empleadoras, lo que pueden utilizar para organizar actividades de vinculación con el mundo del trabajo o ferias laborales, por ejemplo, así como publicar anuncios relacionados con oportunidades de trabajo como empleadores.

En lo referente a este componente, el personal encargado del sistema es responsable de ejecutar las siguientes acciones:

- Desarrollo y ejecución de pruebas, así como soporte técnico necesario para la plataforma de intermediación laboral con cobertura institucional.

- Elaboración de un manual de procedimientos de la plataforma. 
- Capacitación y asesoramiento a las sedes, unidades académicas y otras instancias universitarias sobre el uso de la plataforma.

- Articulación con las instancias encargadas de cursos de actualización profesional que pueda generar un impacto positivo en el desempeño de las personas graduadas.

- Establecimiento de una estrategia institucional de asesoramiento para la búsqueda de empleo, dirigida a personas graduadas y estudiantes.

- Implementación de una metodología de acompañamiento en la inserción laboral de la persona con alguna discapacidad.

- Creación de un mecanismo de divulgación de la plataforma con la comunidad universitaria y sector empleador.

- Elaboración de instrumental para evaluar el asesoramiento brindado y el alcance de la plataforma, así como insumos de apoyo a los procesos de diseño curricular y gestión de la calidad de la oferta formativa de la institución.

b) Análisis contextual del trabajo profesional

Se basa principalmente en la consulta, interpretación, desarrollo y promoción de los estudios de seguimiento, tanto a personas graduadas como con organizaciones que las puedan contratar. $\mathrm{Al}$ respecto se puede determinar que este tipo particular de investigaciones se enfocan en tres grandes aspectos: el proceso y las condiciones de inserción laboral de las personas profesionales, los criterios sobre la formación universitaria con respecto al desempeño laboral, y las necesidades de actualización profesional o especialización; dichos estudios aportan al mejoramiento de las carreras y su gestión, relacionados desde sus inicios con los procesos de autoevaluación y acreditación, porque permiten valorar la calidad de la formación mediante su desempeño profesional, así como las oportunidades de mejora de los planes de estudio como lo señalan Lobo, Vargas, Gamboa, González, y Morúa (2009).

Este análisis contextual requiere una visión de la inserción laboral como la que propone Rivas (2003) al argumentar que es un proceso complejo que debe analizarse desde un nivel macro que comprende las condiciones del mundo laboral y las características de las personas como talento humano, así como desde el nivel micro que se enfoca en las experiencias de socialización laboral de la persona y las organizaciones en las que se viven. Especialmente para las y los profesionales universitarios es fundamental que el puesto de trabajo se relacione estrechamente con la carrera de la que se titularon, lo cual condiciona directamente su adaptación al cargo y por ende a la organización, además de asegurar una inserción exitosa y favorecer su satisfacción laboral, mediante el desempeño de su profesión.

Estos razonamientos dan fundamento a este segundo componente del modelo, desde el cual se propone coordinar con el OLaP-CONARE, para obtener información estratégica sobre las carreras de la universidad, que permita no solamente una toma de decisiones informada y contextuada, sino que además oriente las acciones en función de estos tres componentes; esto en apego a lo estipulado en la Carta Constitutiva del propio OLaP (CONARE, 2008).

Además de la información que se extrae de los estudios de la OLaP, cada universidad requiere ampliar y profundizar en dichos datos, en procura además de conocer la incidencia o impacto de sus carreras en el país, mediante la incorporación al tejido sociolaboral de sus profesionales como agentes de cambio, por lo que es necesario efectuar sus propias investigaciones o consultas al respecto, para lo cual se proponen las siguientes acciones:

- Desarrollar sistemas de información institucional que permitan cruzar diferentes bases de datos permitiendo, no solamente tener mayor cantidad de datos por persona graduada, sino que además facilite hacer análisis estratégico para la gestión de la docencia, en la línea de Learning analytics o de inteligencia de negocios como se denomina en la empresa. 
- Elaborar periódicamente estudios de seguimiento a personas graduadas y organizaciones empleadoras, así como de prospección de empleo por carrera, los cuales permiten detectar en cada momento la situación del mercado laboral, los sectores que están generando espacios de trabajo, los perfiles y los requerimientos de las organizaciones en los diferentes ámbitos.

- Promoción y asesoramiento a las unidades académicas y sedes regionales para que realicen sus propios estudios, ya sea en el marco de los procesos de autoevaluación, acreditación o diseño curricular, así como uno de los ejes de investigación para los trabajos finales de graduación, o proyectos a cargo del personal académico de la institución.

c) Asesoría para la vinculación con el mundo del trabajo.

El tercer componente se basa en el enfoque de Orientación Laboral que propone el SIOIE (2010b) con el cual se busca que las personas analicen su situación laboral, decidan sus proyectos ocupacionales y gestionen con autonomía la planificación de sus objetivos laborales. Para ello se desarrollan en dos líneas, la primera de ellas consiste en una serie de estrategias para orientar en forma colectiva al estudiantado y a las personas graduadas para su inserción al mundo del trabajo laboral, tales como:

- Charlas de preparación sobre plan de búsqueda de empleo, currículum vitae profesional y entrevista laboral.

- Elaboración y publicación de productos multimedia con temas similares a las charlas, para que la persona pueda utilizarlo en cualquier momento.

- Foros para la comunidad universitaria sobre temas como el emprendedurismo y los procesos de contratación de instituciones estatales.

La segunda vía de asesoramiento sobre buenas prácticas de vinculación con el mundo del trabajo va dirigida a las unidades académicas, sedes regionales y otras instancias universitarias, por ejemplo:

- Reuniones de prospección con organizaciones empleadoras, para valorar oportunidades de articulación, programas de pasantías laborales (que incluso podrían curricularizarse como plan piloto) o de práctica supervisada.

- Feria de vinculación laboral: para favorecer el contacto inicial y la coordinación de las instancias universitarias con las organizaciones (estatales o privadas) empleadoras, sin la pretensión de generar expectativas de contratación como sucede con las ferias de empleo.

Ambas vías de asesoramiento se nutren de la información que se genera con los estudios de seguimiento (Gutiérrez et al., 2018), de los cuales se logra extraer una serie de requerimientos que el trabajo exige a las personas profesionales, relacionadas con experiencias laborales, manejo de nuevas tecnologías y competencias, actitudes de adaptación a las actuales formas de trabajo, entre otras. Ante este panorama, el perfil profesional se convierte en el principal (y muchas veces el único) archipiélago de certeza con el que cuenta la persona graduada para aprender a navegar en el mar de incertidumbres (Morin, 1999). Con el asesoramiento que se propone se busca que la persona sea capaz de transformar cada una de estas incertidumbres en una nueva certeza profesional, aprovechando las oportunidades de actualización a su alcance.

A su vez, dichas incertidumbres deben ser consideradas no solamente en su plan de búsqueda de empleo, sino que también, en el diseño, planificación y gestión curricular, en procura de la pertinencia social de los planes de estudio y de su mejoramiento permanente. 


\section{CONCLUSIONES Y RECOMENDACIONES}

Del presente análisis se puede concluir que las personas estudiantes y graduadas universitarias también se ven afectadas por la incertidumbre (Morin, 1999) que les genera una desmesurada sobreoferta de profesionales, como lo demuestra el Programa Estado de la Nación (2019), en ocasiones con un perfil muy similar o con un considerable desfasaje con los requerimientos del mundo laboral actual según Gutiérrez et al. (2019) y Teichler (2005), frente a un panorama global y local con indicadores laborales poco alentadores como los descritos por el INEC (2020), Gutiérrez et al. (2018) y Gutiérrez et al. (2019), y cuya mejoría dista aún de manifestarse, teniendo como consecuencia un ascenso en las tasas de presión general, de desempleo, de subempleo y de poca relación entre carrera y trabajo, así como una marcada asimetría por género en el acceso a las oportunidades en el trabajo.

Debido a la complejidad del fenómeno de inserción laboral, se puede asegurar que una sola disciplina resulta insuficiente para su investigación-intervención (González, 2019), ya que se requiere de una visión interdisciplinaria y multisectorial para su abordaje complejo; es por ello que se estima fundamental la organización de un equipo de trabajo que sume, al menos, un tiempo completo de dedicación (aproximadamente 40 horas semanales), lo que según De Pablo (2011), se requiere para atender los requerimientos básicos de estas instancias. En Costa Rica, según González (2019), estos servicios son dirigidos por profesionales con formación en disciplinas como Administración, Educación, Orientación, Psicología e Informática.

Los servicios de empleo de las instituciones de educación superior no solamente deben facilitar la vinculación de la oferta y la demanda, es decir, las personas estudiantes y graduadas que requieren empleo y las organizaciones que requieren talento profesional cualificado, sino que además, necesitan generar su propio análisis contextual periódicamente, con lo que se puede favorecer en gran medida, el asesoramiento laboral tanto para las personas oferentes y organizaciones demandantes, como a las instancias universitarias encargadas de la gestión de las carreras y la conducción superior institucional, de la manera en que se plantea en la presente propuesta de modelo de vinculación laboral, elemento diferenciador con respecto a los servicios de empleo estudiados, así como el abordaje integral y estratégico para facilitar información pertinente y actualizada para la toma de decisiones de conducción superior institucional, resultante en gran medida de las alianzas estratégicas con otros sectores como las organizaciones del mundo laboral, como recomienda Barreto (2016) y como se ilustra en la Figura 1.

Considerando lo anterior, se comprende la necesidad de que las universidades desarrollen sus propios estudios de seguimiento a personas graduadas y organizaciones empleadoras, que complementen, profundicen y actualicen los resultados que aporta el OLaP desde la perspectiva de Gutiérrez et al. (2018) y Gutiérrez et al. (2019), permitiéndoles conocer sobre el proceso de inserción laboral, la pertinencia de su oferta educativa y necesidades de formación continua para favorecer su desarrollo profesional a lo largo de la vida, la gestión académica de las carreras, así como la toma de decisiones institucional con respecto a la incidencia o impacto que tiene en el desarrollo nacional y bienestar social, especialmente desde la inserción de sus profesionales en diferentes ámbitos de trabajo donde se espera que sean agentes de cambio.

Además, con este enfoque se aporta considerablemente a lo estipulado en el Plan Nacional de la Educación Superior Universitaria Estatal 2016-2020 de CONARE, en relación con el seguimiento a la inserción laboral de las personas egresadas, y en mayor detalle como insumo importante en los requerimientos que exige el SINAES (2009) para los procesos de autoevaluación, acreditación y mejoramiento de calidad de las carreras:

- Razones de una posible prolongación del tiempo de estudios y graduación.

- Información actualizada de las condiciones de mercado de trabajo de la disciplina e inserción laboral, además del ajuste a la realidad sociolaboral.

- Índice de satisfacción con la formación recibida por las personas graduadas. 
- Conocimiento y oferta académica para las necesidades de actualización profesional.

- Actividades de vinculación con las personas profesionales que egresan.

De la misma forma, se estima que con la implementación de este modelo se favorece la calidad de la formación y la vinculación con las organizaciones empleadoras, tanto del sector público como privado, atendiendo las recomendaciones expuestas por organismos internacionales como IESALC (2018) y la propia OIT en Muñoz (2012), lo que permitiría valorar el aporte de dicho modelo en la gestión de las universidades que consideren implementar algunas líneas de acción descritas en el presente diseño.

\section{REFERENCIAS BIBLIOGRÁFICAS}

Agencia Nacional de Empleo (2020). ¿Qué es la Agencia Nacional de Empleo? Recuperado de https://www.ane.cr/H ome/Acerca

Barreto, A. (2016). Orientación Laboral Innovadora y Desarrollo de Carrera: Contexto Venezolano. Revista cuatrimestral de la Red cubana de alternativas en Psicología, 4 (10), 89-95. Recuperado de http://acupsi.org/nu mero/20/vol-4-nm-10-2016.html

Consejo Nacional de Rectores [CONARE]. (2008). Carta constitutiva del proyecto Observatorio Laboral de Profesionales. San José, Costa Rica: CONARE.

De Pablo, J.M. (2011). Desconcentración y descentralización de los Servicios Públicos de Empleo en Centroamérica y República Dominicana. Revista Trabajo, 24, 37-59. Recuperado de http://rabida.uhu.es/dspace/handle/102 $72 / 5758$

Echeverría, B., Isus, S., Martínez, M. y Sarasola, L. (2008). Orientación Profesional. Barcelona: OUC.

Enriques, M. (2007). Desarrollo y gestión de carreras con adultos en el siglo XXI: lecturas hacia una armonización de lo global y de lo individual. Electronic Journal of Research in Educational Psychology, (5), 75-101. Recuperado de http://www.redalyc.org/articulo.oa?id=293121941006

González, F. (2019). Modelos de intervención de los servicios de empleo en las universidades públicas costarricenses: una caracterización teórica desde la orientación laboral (Tesis de maestría). Recuperado de http://kerwa.ucr.ac.cr/h andle/10669/79186

Gutiérrez, I., Kikut, L., Corrales, K. y Picado, C. (2018). Seguimiento de la Condición Laboral de las Personas Graduadas 2011-2013 de las Universidades Costarricenses. San José, Costa Rica: CONARE. Recuperado de https://bit.ly/ 3 f1Stv0

Gutiérrez, I., Madrigal, O., Corrales, K. y Kikut, L. (2019). Empleadores 2016 de personas graduadas de universidades estatales. San José, Costa Rica: CONARE. Recuperado de https://bit.ly/39v3l3p

Instituto Internacional de la UNESCO para la Educación Superior de América Latina y el Caribe [IESALC]. (2018). Plan de Acción 2018-2028, III Conferencia Regional de Educación Superior (CRES). Recuperado de https://ww w.iesalc.unesco.org/2018/12/13/informe-general-de-la-cres-2018/

Instituto Nacional de Estadística y Censo [INEC]. (2016). Encuesta Continua de Empleo, IV Trimestre de 2016. Recuperado de https://www.inec.cr/sites/default/files/documetos-biblioteca-virtual/reempleoece2016_ivt.pd $\mathrm{f}$

Instituto Nacional de Estadística y Censo [INEC]. (2020). Encuesta Continua de Empleo, I Trimestre de 2020. Recuperado de https://bit.ly/3f6daGt

Lobo, N., Vargas, A., Gamboa, O., González, R. y Morúa, M. (2009). Estudio de seguimiento de personas graduadas del periodo 2000-2006 de once programas de posgrado de las Universidades Estatales Costarricenses. San José, Costa Rica: CONARE.

Mazza, J. (2003). Servicios de Intermediación Laboral: enseñanzas para América Latina y el Caribe. Revista de la CEPAL, (80), 165-183. Recuperado de https://repositorio.cepal.org/handle/11362/10902

Morín, E. (1999). Los siete saberes necesarios para la educación del futuro. París, Francia: UNESCO 
Franky González Conejo. Diseño de un modelo universitario de vinculación laboral y apoyo a la GeSt...

Muñoz, P. (20 de abril, 2012). Generaciones de jóvenes profesionistas, en riesgo de caer en la economía informal: OIT. Periódico La Jornada, p 1. Recuperado de http://www.jornada.unam.mx/2012/04/20/sociedad/045n 1soc

Organización Internacional del Trabajo [OIT]. (2020). Perspectivas sociales y del empleo en el mundo - Tendencias 2020. Recuperado de https://bit.ly/32Tquev

Programa Estado de la Nación (2019). VII Estado de la Educación. San José, Costa Rica: Programa Estado de la Nación.

Rivas, F. (2003). Asesoramiento vocacional: Teoria, práctica e instrumentación. España: Ariel Psicología.

Sistema Nacional de Acreditación de la Educación Superior [SINAES]. (2009). Manual de acreditación oficial de carreras de grado. San José, Costa Rica: SINAES.

Sistema Nacional de Intermediación, Orientación e Información de Empleo [SIOIE]. (2010a). Guía para la Gestión de la Orientación Laboral. San José, C.R: INA, MEP, MTSS.

Sistema Nacional de Intermediación, Orientación e Información de Empleo [SIOIE]. (2010b). Manual del Servicio Público de Empleo de la República de Costa Rica. San José, C.R: INA, MEP, MTSS. Recuperado de http://es.scr ibd.com/doc/96459/Sistema-Nacional-de-Intermediacion-Orientacion-e-Informacion-de-Empleo-SIOIE

Teichler, U. (2005). Graduados y empleo: investigación metodológica y resultados. Los casos de Europa, Japón, Argentina y Uruguay. Buenos Aires: Miño y Dávila srl.

\section{Notas}

[1] Debido a que el autor es uno de los dos funcionarios a cargo del servicio de intermediación laboral en la Universidad Nacional.

\section{INFORMACIÓN ADICIONAL}

Cómo citar: González Conejo, F. (2021). Diseño de un modelo universitario de vinculación laboral y apoyo a la gestión académica. Revista Educación, 45(2). Recuperado de http://doi.org/10.15517/revedu.v45i1.43 083 